\title{
Application of software tools for symbolic description and modeling of mechanical systems
}

\author{
Andrei Banshchikov, Alexander Vetrov \\ Matrosov Institute for System Dynamics and Control Theory of Siberian Branch of Russian \\ Academy of Sciences, Irkutsk, Russia \\ E-mail: bav@icc.ru
}

\begin{abstract}
The paper presents two software tools (graphical editor and software package). The editor is designed for the formation of a symbolic description of a mechanical system using the Lagrange formalism. A system of the absolutely rigid bodies connected by joints is considered as a mechanical system. The editor is a user interface by which one sets the structure of the interconnection of bodies (system configuration) as well as the geometric and kinematic characteristics for each body of the system. The created structure and the entered data are automatically presented in the form of a text file, which is used as an input file for the software package for modeling mechanical systems in a symbolic form with a computer. The use of these software tools is shown in detail in the example of the analysis of the dynamics of a satellite with a gravitational stabilizer in a circular orbit. For this system, the kinetic energy and force function of an approximate Newtonian gravitational field were obtained, nonlinear and linearized equations of motion were constructed, and the question of the stability of the relative equilibrium position was considered.
\end{abstract}

\section{Introduction}

In recent years, the importance of computer algebra systems (CAS) $[1,2]$ and the methods of their use [3] for scientific computations in various fields of natural sciences and engineering knowledge has grown significantly. Many years of experience of the authors [4] show that CASs turned out to be quite effective at the stages of constructing mathematical models and analyzing their properties. The combination of methods of analytical mechanics and the theory of stability of motion with the algorithms of CAS allows one to create quite powerful tools for the study of mechanical systems.

The characteristic feature of our investigations is that practically all the computations on a computer are conducted in symbolic (analytical) form. When it is difficult to conduct research exclusively in symbolic form, one can proceed to "digitizing" some (or all) of the parameters of the problem under consideration and, thereby, close the question of rounding errors. This allows the researcher to penetrate into the qualitative nature of the processes as deeply as possible and move on to a quantitative (numerical) assessment at a later stage.

As can be seen from the publications, more often there is an approach to using the CAS as a calculator for solving a particular problem. There is another approach when, based on

Copyright (C) 2020 for this paper by its authors. Use permitted under Creative Commons License Attribution 4.0 International (CC BY 4.0). 


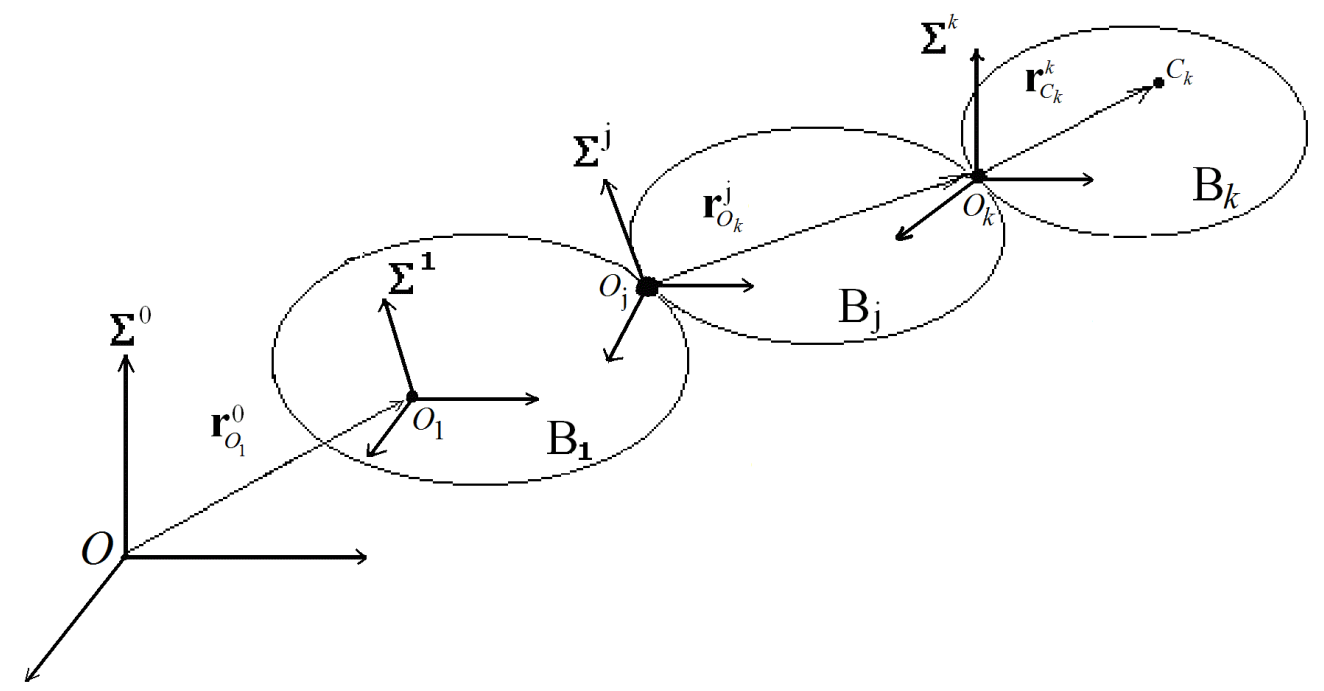

Figure 1. The structure of interconnection between the bodies.

the internal programming language of CAS (in our case - "Mathematica" [5]), a software is developed to solve a specific class of problems.

Some algorithms for analyzing the dynamics and stability of motion of mechanical systems using symbolic computations are presented in [6,7]. The application of computer algebra methods to the problems of celestial mechanics has rich history and till today attracts academic attention (see, for example, $[8,9]$ ).

\section{Graphic editor for the description of mechanical systems}

As a mechanical system, a system of the absolutely rigid bodies $\mathrm{B}_{i}(i=\overline{1, N})$, connected by one-two-three-degree joints is considered. The body $\mathrm{B}_{1}$ is a carrier. Its position is determined relative to the inertial coordinate system (CS) $\Sigma^{0}$. Let us associate the coordinate system $\Sigma^{1}$ to the rigid body $\mathrm{B}_{1}$. The starting point of that system will be at the point $O_{1}$. The bodies are connected so that for each $\mathrm{B}_{k}$ there is a common point $O_{k}\left(O_{k} \in \mathrm{B}_{k}, O_{k} \in \mathrm{B}_{j}, j=\overline{1, N}\right.$, $k=\overline{2, N}, j<k$ ) (see Figure 1), or joints allow translational displacements $\mathrm{B}_{k}$ relative to $\mathrm{B}_{j}$. With the body $\mathrm{B}_{k}$ in a point $O_{k} \in \mathrm{B}_{k}$ we will associate a CS $\Sigma^{k}$. The angular position of $\Sigma^{k}$ relative to $\Sigma^{j}$ is determined by the matrix $\Re^{j k}$, which elements are the functions of the selected generalized coordinates.

It is assumed that the system is under the influence of forces of potential and non-potential nature.

In accordance with the configuration of the mechanical system, the number of bodies $N$ in the system is defined, and for each body $\mathrm{B}_{k}(k=\overline{1, N})$ are specified:

- $j$ is the number of body $\mathrm{B}_{j}$, to which body $\mathrm{B}_{k}$ is connected; or the coordinate system number $\Sigma^{j}$ with respect to which the position of the body $\mathrm{B}_{k}$ is determined $(j<k$ for $k>1$ and $j=0$ for the body $\mathrm{B}_{1}$ ),

- the numbers of rotation axes and its corresponding identifiers of rotation angles,

- $\mathbf{r}_{O_{k}}^{j}$ is the radius vector of point $O_{k}$ of connection of bodies $\mathrm{B}_{j}$ and $\mathrm{B}_{k}$ in the axes $\Sigma^{j}$,

- $\mathbf{r}_{C_{k}}^{k}$ is the radius vector of the mass center of the body $\mathrm{B}_{k}$ in the axes $\Sigma^{k}$,

- $\mathbf{V}_{O_{k}}^{j}$ is the vector of relative linear velocity of point $O_{k}$ in the projections onto axes $\Sigma^{j}$ (for the body $\mathrm{B}_{1}$ is the vector of absolute linear velocity), 
- the body mass $m_{k}$ and its tensor of inertia $\boldsymbol{\Theta}_{O_{k}}$ relative to the point $O_{k}$,

- the list of generalized coordinates.

In the integrated development environment Embarcadero Delphi using the programming language Object Pascal, a graphical editor was created. The software was created within the classical paradigm of object-oriented programming using an incremental development model. The editor is intended for the formation of a symbolic description of the studied mechanical system using the Lagrange formalism. As an example, we will consider the description of a satellite with a stabilizer in the paragraph 3 .

The user interface of the editor allows

1) Calling the dialog box for a general description of the mechanical system and filling in the form, indicating, for example, the type of potential forces influencing the system,

2) Defining the structure of the interconnection of bodies (system configuration) using editor tools,

3) Assigning semantic data (geometric and kinematic characteristics) to the bodies. In order to set the semantic data, the context menu is used. These characteristics include, for example, the radius vectors of connection points of the bodies and the vector of relative linear velocities of these points; the radius vectors of the centers of mass; the numbers of the rotation axes and their corresponding rotation angle identifiers; and a list of generalized coordinates,

4) Generating automatically a symbolic description of the investigated mechanical system.

The final description is saved as a text file and served as an input file for another software package for modeling and qualitative analysis of mechanical systems in a symbolic form.

\section{Description of a satellite with a gravitational stabilizer}

The mass center (point $O_{1}$ ) of the system moves along the circular Keplerian orbit of radius $R$ with constant angular velocity. The stabilizer is a rigid rod with point mass at its free end. The rod is connected to the satellite at point $\mathrm{O}_{3}$ with a 2-degree-of-freedom suspension (see Figure 2). Here $l>0$ is the rod length; $r \geq 0$ is the distance from the system mass center to the point of attachment of the rod. The system is influenced by a gravitation moment.

So, for the system under scrutiny (Figure 2), input data assigning the geometric and kinematic characteristics have the following form:

The number of bodies is 4 .

Body 1 (orbital coordinate system $O_{1} x_{1} y_{1} z_{1}$ ) is connected to the body numbered as 0 (inertial coordinate system $\Sigma^{0}$ or $\left.\bar{O} x_{0} y_{0} z_{0}\right)$. The mass and the inertia tensor are zero.

The numbers of the axes of rotation are $\{3,0,0\}$ and angles of rotations $\{-\chi, 0,0\}$, i.e. the orientation of the orbital coordinate system with respect to the inertial coordinate system is determined by one rotation at the angle $-\chi$ about the third axis $\bar{O} z_{0}$. Zeros among the numbers of the rotation axes indicate that there are no rotations about other axes.

The radius vector of the point $O_{1}$ in the coordinate system $\Sigma^{0}$ is $\mathbf{r}_{O_{1}}^{0}=(R \sin \chi, R \cos \chi, 0)$. The radius vector of the body mass center is $\mathbf{r}_{C_{1}}^{1}=(0,0,0)$. The vector of relative linear velocity of point $O_{1}$ is $\mathbf{V}_{O_{1}}^{0}=\left(R \omega_{0} \cos \chi,-R \omega_{0} \sin \chi, 0\right)$, where $\omega_{0}=\dot{\chi}=$ const is the module of orbital angular velocity.

Body 2 (satellite) is described with respect to Body 1 . The body mass is $M_{2}$.

The numbers of rotation axes are $\{2,3,1\}$ and the respective angles of rotations are $\{\psi, \theta, \varphi\}$, i.e. the orientation of the satellite with respect to the orbital coordinate system is determined by the sequence of three rotations: about the second axis $O_{1} y_{1}$ at an angle $\psi$; about the third axis $O_{1} z_{1}$ at the angle $\theta$; about the first axis $O_{1} x_{1}$ at the angle $\varphi$. The radius vector of the point $O_{2}$ with respect to the orbital coordinate system is $\mathbf{r}_{O_{2}}^{1}=(0,0,0)$, i.e. points $O_{1}$ and $O_{2}$ coincide. The radius vector of the mass center of satellite is $\mathbf{r}_{C_{2}}^{2}=(0,0,0)$, i.e. the satellite mass center is at point $O_{1}$. The vector of relative linear velocity of point $O_{2}$ is $\mathbf{V}_{O_{2}}^{1}=(0,0,0)$. 


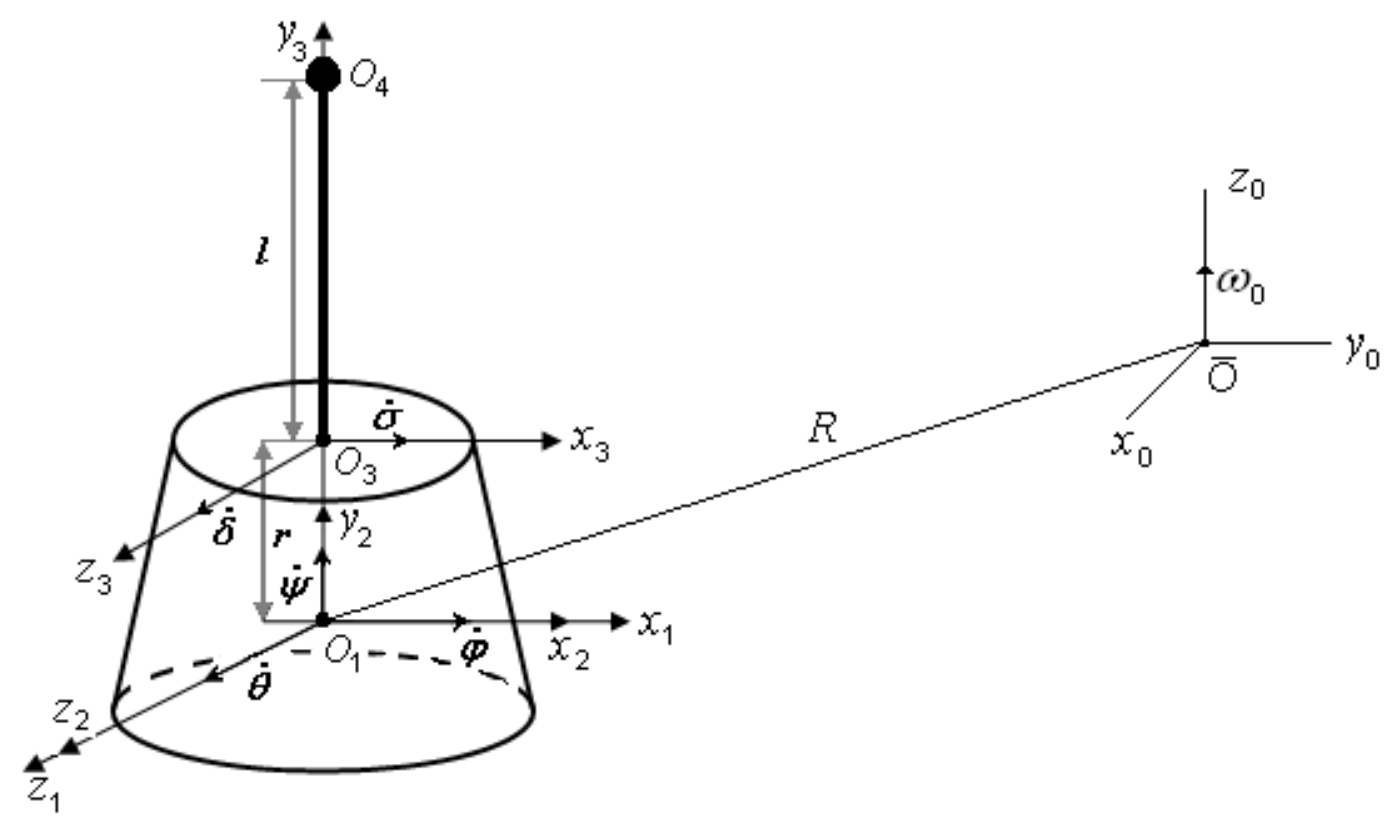

Figure 2. The satellite with a stabilizer.

The body's tensor of inertia at point $O_{2}$ is written as $\Theta_{O_{2}}=\left(\begin{array}{ccc}J_{x} & 0 & 0 \\ 0 & J_{y} & 0 \\ 0 & 0 & J_{z}\end{array}\right)$, i.e. $J_{x}, J_{y}, J_{z}$ are satellite principal inertia moments.

Body 3 (rod) is connected to Body 2. The numbers of rotation axes are $\{3,1,0\}$ and the angles of rotations are $\{\delta, \sigma, 0\}$, i.e. orientation of the body with respect to the coordinate system $O_{1} x_{2} y_{2} z_{2}$ associated with the satellite is determined by the sequence of two rotations: about the third axis at an angle $\delta$ and about the first axis at an angle $\sigma$.

The radius vector of the point $O_{3}$ of the connection of the bodies is $\mathbf{r}_{O_{3}}^{2}=(0, r, 0)$. The radius vector of the mass center is $\mathbf{r}_{C_{3}}^{3}=(0, l / 2,0)$. The vector of relative linear velocity of the point $O_{3}$ is $\mathbf{V}_{O_{3}}^{2}=(0,0,0)$.

The body inertia tensor at point $O_{3}$ is written as $\boldsymbol{\Theta}_{O_{3}}=\left(\begin{array}{ccc}m l^{2} / 3 & 0 & 0 \\ 0 & 0 & 0 \\ 0 & 0 & m l^{2} / 3\end{array}\right)$, where $m$ is the mass of rod.

Body 4 (the point of the mass $m_{0}$ on rod) is connected to Body 3. The point inertia tensor is zero. The radius vector of the point $O_{4}$ of the joint of the bodies, its relative linear velocity, and the radius vector of the center of mass, respectively, have the following form:

$$
\mathbf{r}_{O_{4}}^{3}=(0, l, 0) ; \quad \mathbf{V}_{O_{4}}^{3}=\mathbf{r}_{C_{4}}^{4}=(0,0,0)
$$

Thus, the described conservative mechanical system has five degrees of freedom (aircraft angles $\psi, \theta, \varphi$ as well as rod rotation angles $\delta, \sigma)$. It is located in the Newtonian field of gravitation to the center of the circle $\bar{O}$.

We have to emphasize that the description of the mechanical system is formed in interactive mode, when the user inputs the above mentioned data into corresponding windows of the editor (see, for example, Figure 3). This important process is conducted just once. Later, it is always possible to correct input data stored in a file (see Figure 4). 


\begin{tabular}{|l|}
\hline Body description (2) \\
Previous body number \\
\hline 1 \\
Body mass \\
\hline M2 \\
Radius vector of connection point \\
\hline $0,0,0$ \\
\hline Radius vector of mass center \\
\hline $0,0,0$ \\
\hline Vector of relative linear velocity \\
\hline $0,0,0$ \\
\hline Numbers of rotation axes \\
\hline $2,3,1$ \\
\hline Rotation angles \\
\hline psi, teta, phi \\
\hline \begin{tabular}{l} 
Tensor of inertia \\
\hline Jx
\end{tabular} \\
\hline $\begin{array}{l}0 \\
0\end{array}$ \\
\hline 0
\end{tabular}

Figure 3. The geometric and kinematic characteristics of the second body.

\section{Construction of a symbolical model for a satellite with a stabilizer}

While constructing the mathematical model for complex mechanical objects, searching for its solutions and conducting the qualitative analysis of their properties, it is necessary to operate with bulky analytical expressions. Thus, it is relevant to apply computer algebra systems and develop specialized software on the basis of these systems. Another software tool used in this paper is designed to model the systems of interconnected absolutely rigid bodies, and also to examine the questions of stability and stabilization of solutions of linearized models on the basis of classical theorems of motion stability. The software package is a set of interactive programs executed in the interpretation mode in the CAS "Mathematica" environment.

By the construction of a symbolical model, one implies the obtaining of nonlinear and linearized differential equations of motion of mechanical systems in symbolic form in computer memory.

Let us present a list of problems solved using the software package for the mechanical system described in paragraph 3.

Problem 1. The rotation axis numbers and the corresponding rotation angle identifiers for each body $\mathrm{B}_{k}$ are used for automatic construction of the

$\triangleright$ Matrix of directional cosines $\Re^{j k}$ which determines the angular position of CS $\Sigma^{k}$ relative to $\Sigma^{j}$

$\triangleright$ Vector of the relative angular velocity $\boldsymbol{\omega}_{k}^{j}$ of the $k$-th body in CS $\Sigma^{j}$ and vector of the absolute angular velocity $\boldsymbol{\omega}_{k}=\boldsymbol{\omega}_{j}+\boldsymbol{\omega}_{k}^{j}$ of the body,

$\triangleright$ Absolute linear velocity vector $\mathbf{V}_{O_{k}}=\mathbf{V}_{O_{j}}+\left[\boldsymbol{\omega}_{k} \times \mathbf{r}_{O_{k}}^{j}\right]+\mathbf{V}_{O_{k}}^{j}$ of point $O_{k}$.

For example, we write out the matrix of directional cosines that determines the angular 


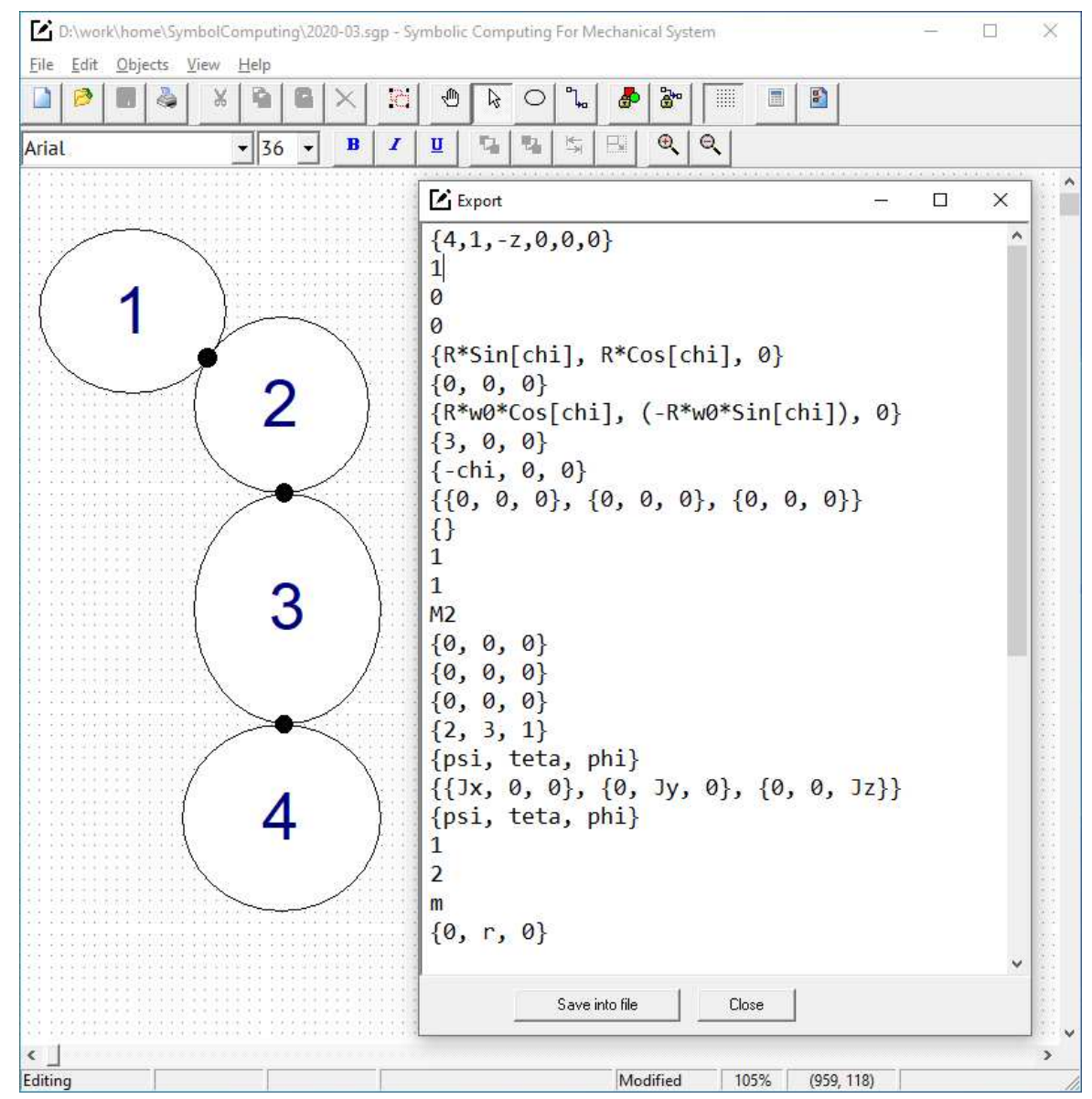

Figure 4. The final symbolic description of a satellite with a stabilizer.

position of the coordinate system $O_{1} x_{2} y_{2} z_{2}$ with respect to the coordinate system $O_{1} x_{1} y_{1} z_{1}$ :

$$
\Re^{12}=\left(\begin{array}{ccc}
\cos \theta \cos \psi & \sin \theta & -\cos \theta \sin \psi \\
\sin \varphi \sin \psi-\cos \varphi \cos \psi \sin \theta & \cos \theta \cos \varphi & \cos \psi \sin \varphi+\cos \varphi \sin \theta \sin \psi \\
\cos \psi \sin \theta \sin \varphi+\cos \varphi \sin \psi & -\cos \theta \sin \varphi & \cos \varphi \cos \psi-\sin \theta \sin \varphi \sin \psi
\end{array}\right)
$$

and the projections of the vector of satellite absolute angular velocity $\boldsymbol{\omega}_{2}$ (of the second body) onto the axes $O_{1} x_{2} y_{2} z_{2}$ connected to the body

$$
\left\{\begin{array}{l}
\omega_{x}=\dot{\varphi}+\dot{\psi} \sin \theta+\omega_{0} \cos \theta \sin \psi \\
\omega_{y}=\dot{\theta} \sin \varphi+\dot{\psi} \cos \theta \cos \varphi-\omega_{0}(\sin \varphi \cos \psi+\sin \theta \cos \varphi \sin \psi) \\
\omega_{z}=\dot{\theta} \cos \varphi-\dot{\psi} \cos \theta \sin \varphi+\omega_{0}(\sin \theta \sin \varphi \sin \psi-\cos \varphi \cos \psi)
\end{array}\right.
$$

Before executing Problem 1, the generalized coordinates are replaced with the corresponding Greek letters, as well as other necessary substitutions are made.

Problem 2. The kinetic energy for the system of bodies as a quadratic form with respect to generalized velocities

$$
T(q, \dot{q})=\frac{1}{2} \sum_{i=1}^{5} \sum_{j=1}^{5} A_{i j}(q) \dot{q}_{i} \dot{q}_{j}+\sum_{i=1}^{5} A_{i}(q) \dot{q}_{i}+A_{0}(q)
$$


and the force function $U(q)$ of the approximate Newtonian field of gravitation have been obtained. Here $q=(\psi, \theta, \varphi, \delta, \sigma)$ is the vector of generalized coordinates. It is established that the rotation angle $\chi$ is a cyclic generalized coordinate, i.e. the system's Lagrangian $L=T(q, \dot{q})+U(q)$ does not depend on this coordinate. The formulas used in solving Problems 2 and 3 are in [4].

Problem 3. Five nonlinear equations of motion in Lagrange form of the $2^{\text {nd }}$ kind have been constructed. Each of equations takes up several screens of the monitor. Therefore, it is impossible to present them in the paper. As a confirmation, let's demonstrate, for example, the terms with respect to the accelerations (the notation $q^{\prime \prime}$, see Figure 5) in the nonlinear equation with respect to the $\theta$ coordinate.

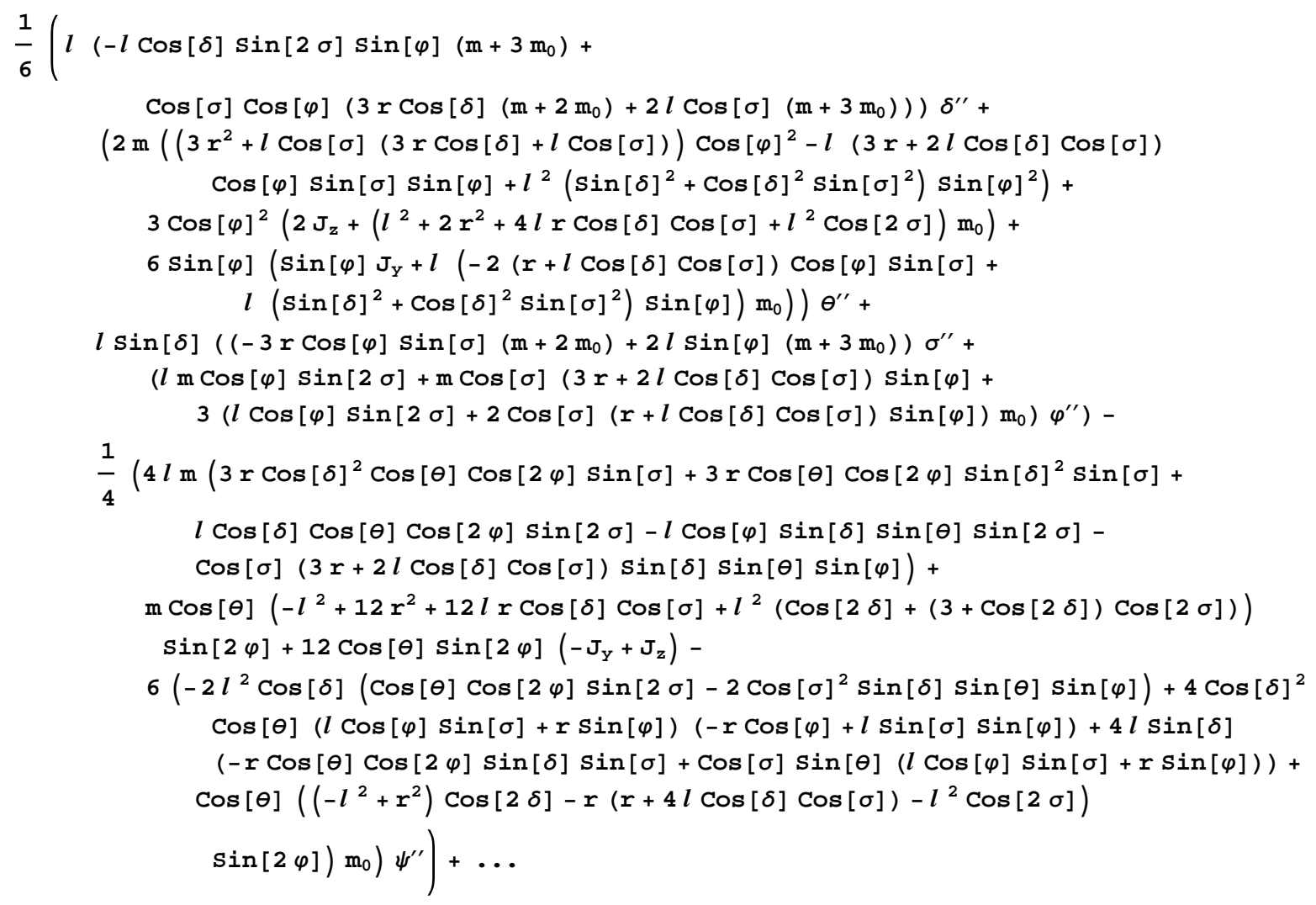

Figure 5. Part of the equation of motion with respect to the $\theta$ coordinate.

Problem 4. Let us assign unperturbed motion in the form

$$
\psi=0, \theta=0, \varphi=0, \delta=0, \sigma=0, \dot{\psi}=0, \dot{\theta}=0, \dot{\varphi}=0, \dot{\delta}=0, \dot{\sigma}=0 .
$$

When moving unperturbed, the system principal central axes of inertia coincide with the axes of orbital coordinate system, and rod is oriented along the radius of the orbit. This is the equilibrium position of a satellite with a stabilizer in regard to orbital coordinate system.

The given unperturbed motion is automatically substituted into nonlinear equations of motion. If motion (2) is the solution, then each equation of motion turns into an identity. Otherwise, the program issues the conditions of existence of a motion, which are to be taken into account by the user in any further computations or which fulfillment has to be ensured.

Problem 5. Constructed are equations of perturbed motion in the first approximation. Linearized in the vicinity of the equilibrium position (2), equations of motion for a satellite with 
a stabilizer are decomposed into two subsystems. Respectively, a "pitch" subsystem $(\theta)$ and a "yaw-and-roll" subsystem $(\psi, \varphi)$ are

$$
\left\{\begin{array}{c}
M_{1} \ddot{q}_{1}+K_{1} q_{1}=0 \\
M_{2} \ddot{q}_{2}+G \dot{q}_{2}+K_{2} q_{2}=0,
\end{array}\right.
$$

where all derivatives are calculated on dimensionless time $\tau=\omega_{0} t$;

$$
\begin{gathered}
q_{1}=\left(\begin{array}{l}
\theta \\
\delta
\end{array}\right) ; \quad q_{2}=\left(\begin{array}{l}
\psi \\
\varphi \\
\sigma
\end{array}\right) ; \quad M_{1}=\left(\begin{array}{cc}
c & f \\
f & d
\end{array}\right) ; \quad K_{1}=3\left(\begin{array}{cc}
b-a & f \\
f & f
\end{array}\right) ; \quad M_{2}=\left(\begin{array}{lll}
a & 0 & 0 \\
0 & b & f \\
0 & f & d
\end{array}\right) ; \\
K_{2}=\left(\begin{array}{ccc}
c-b & 0 & 0 \\
0 & 4(c-a) & 4 f \\
0 & 4 f & 3 f+d
\end{array}\right) ; \quad G=\left(\begin{array}{ccc}
0 & c-b-a & 0 \\
a+b-c & 0 & 0 \\
0 & 0 & 0
\end{array}\right) .
\end{gathered}
$$

Here, we introduce the following notations:

$$
\begin{aligned}
& a=J_{y} ; \quad b=J_{x}+m r(l+r)+\frac{1}{3} m l^{2}+m_{0}(l+r)^{2} ; \quad c=b+J_{z}-J_{x} \\
& d=\left(\frac{m}{3}+m_{0}\right) l^{2} ; \quad f=\left(\frac{m}{2}+m_{0}\right) r l+d ; \quad c-b-a=J_{z}-J_{x}-J_{y} .
\end{aligned}
$$

We can also derive linear equations of motion from the Lagrangian of the system which is expanded in a Taylor series in the vicinity of the solution up to the 2nd order of smallness with respect to deviations of coordinates $\Delta q=q-q^{o}$ from their values in the unperturbed motion. Namely, taking into account the Lagrangian structure (1), the following coefficients are expanded in a series and calculated in the vicinity of equilibrium (2): those with the second degrees of generalized velocities $A_{i j}(q)$ are up to a constant; those with the first degrees of velocities $A_{i}(q)$ are up to linear values with respect to deviations; those with zero degrees of velocities $A_{0}(q)+U(q)$ are up to quadratic components with respect to deviations. This procedure has been called "quadratization" of the Lagrangian.

In the process of simplification of the expression of "quadratized" Lagrangian we have taken into account the condition of circular orbit $\nu / R^{3}=\omega_{0}^{2}$, where, we have to recall, $R$ is the orbit's radius, $\omega_{0}$ is a constant value of orbital angular velocity and $\nu$ is the gravitation constant.

\section{Necessary conditions of stability of equilibrium position}

Equations (3) may be interpreted as equations of oscillations of a mechanical system influenced by potential (with the matrices $K_{1}, K_{2}$ ) and gyroscopic (with the matrix $G$ ) forces. These forces are determined by gravitation forces as well as by orbital motion. The matrices $M_{1}, M_{2}$ play the role of diagonal blocks of a positive definite matrix of kinetic energy.

We introduce the following four dimensionless parameters:

$$
\alpha=\frac{c-b}{a}=\frac{J_{z}-J_{x}}{J_{y}} ; \quad \gamma=\frac{b-a}{c} ; \quad p_{1}=\frac{d}{f} ; \quad p_{2}=\frac{f}{c} .
$$

The physically obtainable values of the parameters are located within the intervals:

$$
-1<\alpha<1, \quad 0<\gamma<1, \quad 0<p_{1} \leq 1, \quad 0<p_{2}<1 .
$$

It is obvious that the characteristic equation of system (3) is factorized as $\Lambda(\lambda) \equiv \Lambda^{(1)} \Lambda^{(2)}=0$. After performing elementary transformations with the characteristic matrices (multiplying their 
rows by positive factors), we obtain the characteristic determinants in notation (4), respectively, in the "pitch" subsystem and in the "yaw-and-roll" subsystem

$$
\begin{aligned}
& \Lambda^{(1)}=\left|\begin{array}{cc}
\lambda^{2}+3 \gamma & p_{2}\left(\lambda^{2}+3\right) \\
\lambda^{2}+3 & \lambda^{2} p_{1}+3
\end{array}\right|=\lambda^{4}\left(p_{1}-p_{2}\right)+3 \lambda^{2}\left(1+\gamma p_{1}-2 p_{2}\right)+9\left(\gamma-p_{2}\right) ; \\
& \Lambda^{(2)}=\left|\begin{array}{ccc}
\lambda^{2}+\alpha & \lambda(\alpha-1) & 0 \\
\lambda(\alpha-1)(\gamma-1) & \lambda^{2}(1+\gamma \alpha)+4(\alpha+\gamma) & \left(\lambda^{2}+4\right) p_{2}(\alpha+1) \\
0 & \lambda^{2}+4 & \lambda^{2} p_{1}+\left(3+p_{1}\right)
\end{array}\right|= \\
& =v_{6} \lambda^{6}+v_{4} \lambda^{4}+v_{2} \lambda^{2}+v_{0}, \quad \text { where } \quad v_{6} \equiv \operatorname{det} M_{2}=(1+\gamma \alpha) p_{1}-(\alpha+1) p_{2} ; \\
& v_{4}=3(1+\alpha \gamma)+(\alpha+1)\left(p_{1}(\alpha+3 \gamma+2)-(\alpha+8) p_{2}\right) ; \\
& v_{2}=(1+3 \gamma+\alpha(\alpha+2 \gamma+3))\left(3+p_{1}\right)+4 \alpha p_{1}(\gamma+\alpha)-8 p_{2}(\alpha+1)(\alpha+2) ; \\
& v_{0} \equiv \operatorname{det} K_{2}=4 \alpha\left((\alpha+\gamma)\left(3+p_{1}\right)-4(\alpha+1) p_{2}\right) \text {. }
\end{aligned}
$$

The characteristic equation of system (3) contains $\lambda$ only in even degrees. The stability of a trivial solution takes place when all roots with respect to $\lambda^{2}$, being simple, will be real negative numbers. The algebraic conditions providing specified properties of roots (necessary conditions of stability), represent the system of inequalities

$$
\begin{aligned}
& \left\{\begin{array}{c}
p_{1}-p_{2}>0, \quad 1+\gamma p_{1}-2 p_{2}>0, \quad \gamma-p_{2}>0, \\
p_{1}^{2} \gamma^{2}+\left(4 p_{2}\left(1-p_{1}\right)-2 p_{1}\right) \gamma+\left(1-4 p_{2}\left(1-p_{1}\right)\right)>0 ;
\end{array}\right. \\
& \left\{\begin{array}{c}
v_{6}>0, \quad v_{4}>0, \quad v_{2}>0, \quad v_{0}>0, \\
D i s \equiv v_{4}^{2} v_{2}^{2}-4 v_{2}^{3} v_{6}-4 v_{4}^{3} v_{0}+18 v_{6} v_{4} v_{2} v_{0}-27 v_{0}^{2} v_{6}^{2}>0 .
\end{array}\right.
\end{aligned}
$$

It is worth noting that the first conditions in (5), (6) is always satisfied by virtue of the positive definiteness of the kinetic energy matrix. The latter inequality in (6) has also been obtained in analytical form and represented in the form of an explicit dependence on the parameters (4). This polynomial is not presented due to being immense.

Emphasize that the calculation of the coefficients $v_{i}(i=\overline{0,3})$ and the discriminant Dis from (6) was also performed using the software package.

A parametric analysis of the stability conditions (5), (6) has been conducted in [10]. This article also considers the possibility of gyroscopic stabilization of an unstable equilibrium position. In contrast to the presented passive stabilization system, the possibility of active control of a gravitational stabilizer is investigated in [11]. Assuming an instability of a potential system, this article considers the problem of stabilization of the system up to asymptotic Lyapunov stability.

\section{Conclusion}

The software allows to automatize, and, consequently, essentially speed up the processes of modeling and dynamic analysis of complicated systems and to avoid errors at all stages of research. The effectiveness of the presented software tools is confirmed, in particular, by their use in the study in symbolic form of (i) the dynamics of a satellite with gyrodines, (ii) the stability of the relative equilibriums of the gyrostat in a circular orbit, (iii) obtaining invariant manifolds and analyzing their stability in the Clebsch-Tisseran-Brun problem [12]. 


\section{Acknowledgments}

The work was supported by the Russian Foundation for Basic Research (grant No. 19-01-00301).

\section{References}

[1] Buchberger B, Collins G E, Loos R and Albrecht R 1983 Computer Algebra: Symbolic and Algebraic Computation (New York: Springer-Verlag)

[2] Davenport J H, Siret Y and Tournier E 1988 Computer Algebra: Systems and Algorithms for Algebraic Computations (New York: Academic Press)

[3] Cohen J S 2003 Computer Algebra and Symbolic Computation: mathematical methods (A K Peters, Ltd.)

[4] Banshchikov A V, Bourlakova L A, Ivanova G N, Irtegov V D, Novickov M A and Titorenko T N 1998 Experience of development and usage of packages of symbolic computations intended for investigation of mechanical systems Elect. Proc. of the 4th Int. IMACS Conf. on Applications of Computer Algebra (Prague: Czech Technical University) URL https://math.unm.edu/ACA/1998/sessions/history/bourlakova/index.html

[5] Wolfram S 1999 The Mathematica Book. Fourth Edition (New York: Cambridge University Press)

[6] Banshchikov A V and Bourlakova L A 1997 Algorithms of Symbolic Computation Used in Stability Analysis Programming and Computer Software 23(3) 173-179

[7] Irtegov V D and Titorenko T N 2001 Using the system "Mathematica" in problems of mechanics Mathematics and Computers in Simulation 57(3) 227-237

[8] Gutnik S A, Guerman A and Sarychev V A 2015 Application of computer algebra methods to investigation of influence of constant torque on stationary motions of satellite Lecture Notes in Computer Science $\mathbf{9 3 0 1}$ 198-209 Springer

[9] Prokopenya A N, Minglibayev M Z and Mayemerova G M 2014 Symbolic calculations in studying the problem of three bodies with variable masses Programming and Computer Software 40(2) 79-85

[10] Banshchikov A V 2002 Parametric analysis of stability conditions for a satellite with a gravitation stabilizer Proc. of the 5th Int. Workshop on Computer Algebra in Scientific Computing ed V Ganzha et al. (Munich: Technische Universität München) pp 1-6

[11] Banshchikov A V 2017 On the Asymptotic Stability of a Satellite with a Gravitational Stabilizer Lecture Notes in Computer Science 10490 16-26 Springer

[12] Irtegov V D and Titorenko T N 2012 Invariant manifolds in the Clebsch-Tisserand-Brun problem Journal of Applied Mathematics and Mechanics 76(3) 268-274 